\title{
Switchable fiber optic laser system for high and low-strain fiber optic sensors remote multiplexing
}

\author{
S. Rota-Rodrigo*a ${ }^{\text {a }}$ L. Rodríguez-Cobo ${ }^{\mathrm{b}}$, M. A. Quintela ${ }^{\mathrm{b}}$, J. M. López-Higuera ${ }^{\mathrm{b}}$, M. López-Amo ${ }^{\mathrm{a}}$ \\ ${ }^{a}$ Department of Electric and Electronic Engineering, Universidad Pública de Navarra, Campus

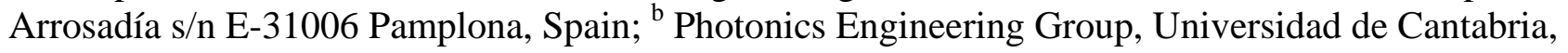 \\ Av. Los Castros s/n, 39005, Santander, Spain
}

\begin{abstract}
In this paper, a $50 \mathrm{Km}$ remote and switchable fiber optic sensing system based on an erbium doped fiber ring laser is proposed. The system allows the integration of different interrogation methods based on the detection of wavelength or amplitude variations of the laser. This integration is carried out by sequentially activating different ring cavities by using an optical switch. With this scheme, the maximum gain zone of the erbium doped fiber amplifier can be used to increase the distance of the sensor system. A bending intensity sensor and a FBG based strain sensor were successfully tested for high and low-strain measurements respectively. This system includes all the active elements inside the monitoring station, enabling the remote monitoring of the sensors.
\end{abstract}

Keywords: Remote fiber optic sensor, strain sensor, erbium doped fiber laser, fiber Bragg grating, sensors multiplexing.

\section{INTRODUCTION}

Optical sensors can provide attractive means to monitor a variety of external perturbations such as: temperature, strain, bend, pressure, and others, due to its high sensitivity, electro-magnetic immunity and compactness. These sensing systems can find important applications in the field of structural health monitoring. [1,2].

Strain measurements are usually performed in structural health monitoring. Depending on the application, low and medium strain measurements are not enough and high strain measurements are needed [3]. Within the fiber optical sensors for strain measurement, two kinds of them are particularly appealing: intensity sensors and wavelength dependent sensors. A number of the intensity ones are based on bending mechanisms, and have been extensively used because they are simple, cheap and reliable. Bending loss technique has also been successfully applied to the measurement of magnitudes such as strain, displacement, pressure, temperature, etc [1-4]. On the other hand, sensors based on Fiber Bragg gratings (FBG) are the most used wavelength selective fiber sensors for strain measurements and offer advantages such as wavelength division multiplexing, good linearity, compactness, a great commercial availability and low cost. [1,2]

Remote sensing has received a higher attention during last years. This technique has been proven as a useful tool for monitoring the optical sensors from a central station located tens kilometers away from the field.[5,6]. For that purpose, multi-wavelength erbium doped fiber ring lasers (MEDFRL) are very attractive sources that provide the required optical power to cover the optical network. Besides, MEDFRL are simple structures with narrow line-width, and compatibility with other optical fiber components [7]. In [8] a combination of an EDFRL with a switch is presented for temperature sensing. However, ring lasers are also susceptible to output power instabilities that can degrade the performance characteristics of the sensor. Different techniques have been proposed to improve the stability like cooling the erbium doped fiber (EDF)[9], combining the erbium doped fiber amplifiers (EDFA) with semiconductor optical amplifiers (SOA) [10] or even with Raman amplification [11]. Other reported possibility is to include a switch to isolate the different channels [8].

*sergio.rota@unavarra.es; phone (0034) 948 169841; fax (0034) 948169720

Fifth European Workshop on Optical Fibre Sensors, edited by Leszek R. Jaroszewicz, Proc. of SPIE Vol. 8794, 87943U · @ 2013 SPIE · CCC code: 0277-786X/13/\$18 · doi: 10.1117/12.2025818 
In this paper we demonstrate a switchable fiber optic laser system for multiplexing remote fiber optic sensors. The system is based on a switchable erbium doped fiber ring laser (EDFRL). The sensors are inside different rings of the EDFRL and are selected sequentially by a switch. Using this technique, no gain competition among different wavelengths as happens in multiwavelength lasers occurs and the utilized wavelength can be always the corresponding to the higher gain of the optical amplifier. The proposed system is able to manage both intensity and FBG based sensors just by selecting the appropriate output channel of the switch. A bending intensity sensor and a FBG sensor have been successfully tested for high and low-strain measurements respectively showing the suitability of the lasing structure.

\section{EXPERIMENTAL SETUP}

The proposed remote sensing system is based on an erbium doped fiber ring laser (EDFRL) with different rings selected by a switch. The gain medium is a commercial EDFA (FIBERAMP-BT 17 by Photonetics). This configuration allows the integration of different interrogation methods based on the detection of wavelength or amplitude variations of the laser. As mentioned above, in this work is presented the multiplexing of a bending intensity sensor and a wavelength strain sensor for high and low-strain measurements respectively. Figure 1 shows the experimental setup of the remote sensing system.

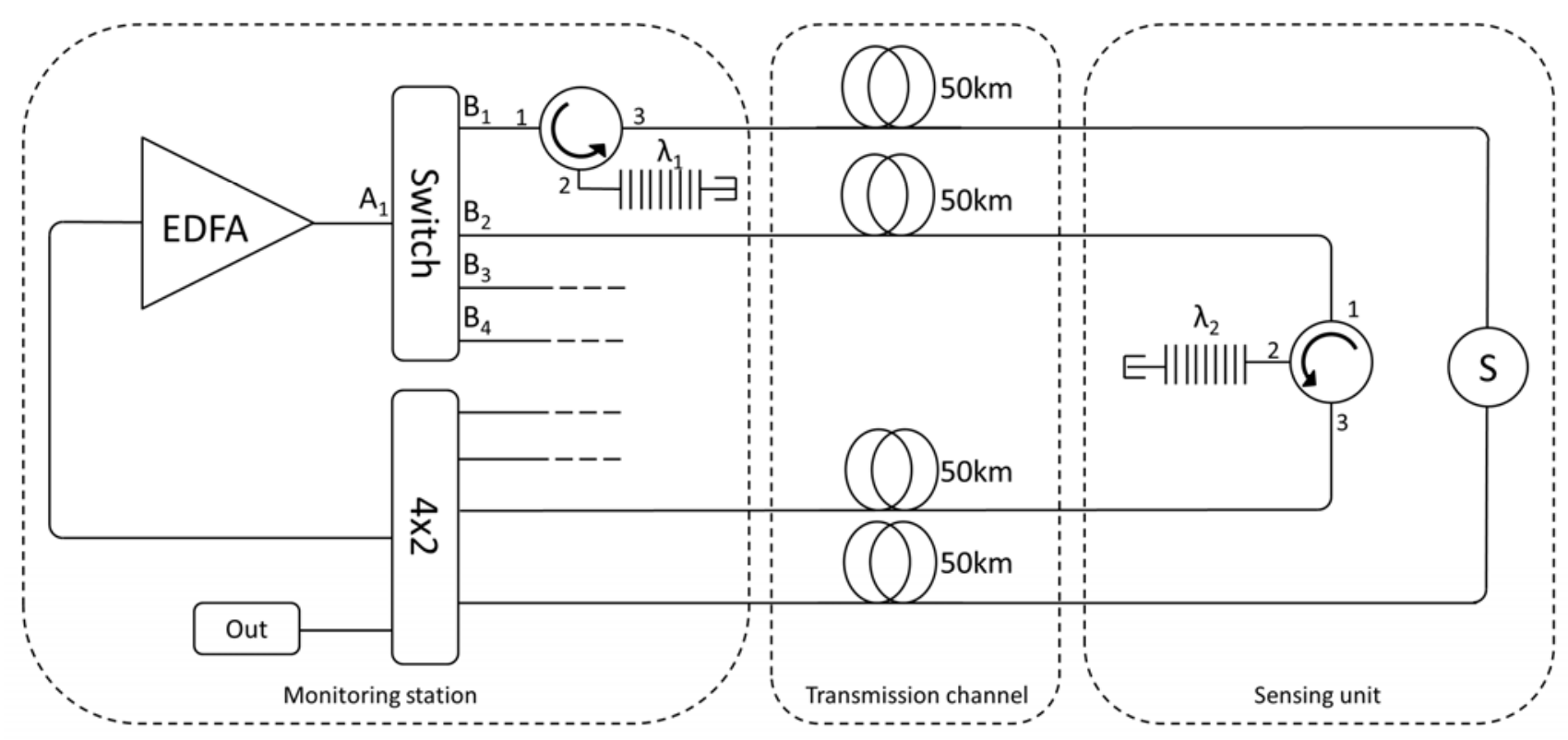

Figure 1. Experimental setup configuration for the EDFRL remote sensing system.

The high-strain EDFRL sensor corresponds to the B1 switch output port. This sensor is based on intensity variations of the laser induced by applying strain to a bending sensor. The wavelength selection is made by an FBG in reflection located at the monitoring station. The FBG are centered at $\lambda 1=1531.66 \mathrm{~nm}$ with a corresponding full-width at half maximum (FWHM) of $0.120 \mathrm{~nm}$ and a reflectivity above $90 \%$. The sensing unit is allocated at $50 \mathrm{Km}$ from the monitoring station and the transmission channel is a cable that includes 4 standard single mode fibers (SMF). In real applications, the cost of the system does not have a significant added increment if several single mode fibers (SMF) are used inside the fiber optic cable instead of only one. The sensing head is made by 20 turns of a SMF around a coil of $38 \mathrm{~mm}$ of diameter. The coil is covered by a rubber tube in order to ensure a uniform response under deformation. The sensor is placed between two anchors, one of them fixed and the other one movable, by using a micrometric step-motor (Fig2). 


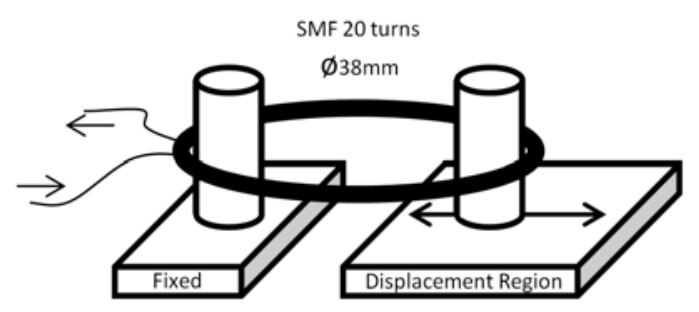

Figure 2. Schematic of the high-strain bending intensity sensor.

The low-strain EDFRL sensor is interrogated using the B2 switch output port. This sensor is made with an in-reflection FBG located at the sensing unit at $50 \mathrm{Km}$ from the monitoring station. When selected, this FBG generates the central lasing wavelength of the EDFRL. The FBG is centered at $\lambda 2=1530.61 \mathrm{~nm}$ with a corresponding FWHM of $0.125 \mathrm{~nm}$ and a reflectivity above $90 \%$. This sensor is based on wavelength variations of the laser induced by applying strain to the FBG.

A $2 \times 4$ switch with a maximum switching time of $370 \mathrm{~ms}$ (HP 86061C) and a $4 \times 2$ coupler are used to select and recombine the different EDFRL sensor signals respectively. The half of the signal is extracted by the coupler to the output and the other half is redirected to the EDFA. The output is connected to an optical spectrum analyzer (OSA, HP70952B) with a resolution of $0.1 \mathrm{~nm}$.

\section{RESULTS}

Firstly, the high-strain sensor was analyzed by selecting the B1 switch output port. In this case an EDFRL at $\lambda 1$ was generated. The amplitude of this lasing wavelength depends on the bending sensor attenuation caused by the strain applied. In order to determinate their response with strain variations, a series of measurements from 0 to 182 mili-strain with steps of 0.13 mili-strain was carried out. Figure 3(a) shows the measured spectra at OSA for the initial position and for the higher strain of 182 mili-strain. The minimum optical signal to noise ratio (OSNR) corresponds to this last position with a value of $46 \mathrm{~dB}$. This obtained SNR is better than the most part of the results from other published remote sensors systems.[13,14]
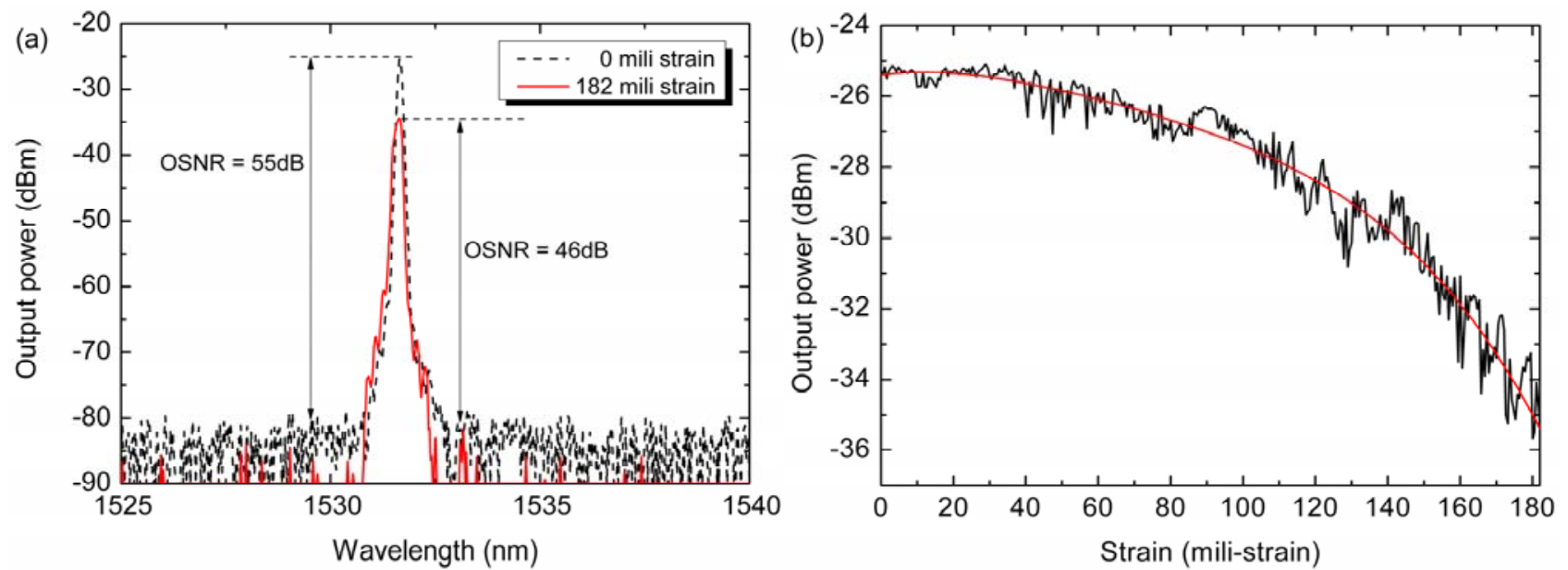

Figure 3. Output spectra of the high-strain sensor for 0 mili-strain (dotted line) and 182 mili-strain (solid line) (a) and, output power evolution with the strain (b).

Figure 3(b) presents the dependence of the output power with stain. That dependence was adjusted by a four degree polynomial curve. The curve slope is determined by the number of sensor turns. We used 20 turns in order to reduce the cladding-modes coupling by curvature effects and to increase the resolution [4]. 
The next step was to carry out the characterization of the low-strain sensor by selecting the B2 switch output port. In this case, the change of the EDFRL wavelength, produced by the strain applied to the FBG, was the parameter to be measured. Figure 4(a) shows the spectrum of four different strain values with an OSNR of $55 \mathrm{~dB}$ for all of them that, again better than the obtained in other published sensors systems.

In order to characterize the sensor with strain, a series of measurements within a range of $3750 \mu \varepsilon$ and steps of $5 \mu \varepsilon$ was performed. Figure 4(b) presents a high linear FBG response against strain with a sensitivity of $0.97 \mathrm{pm} / \mu \varepsilon$.
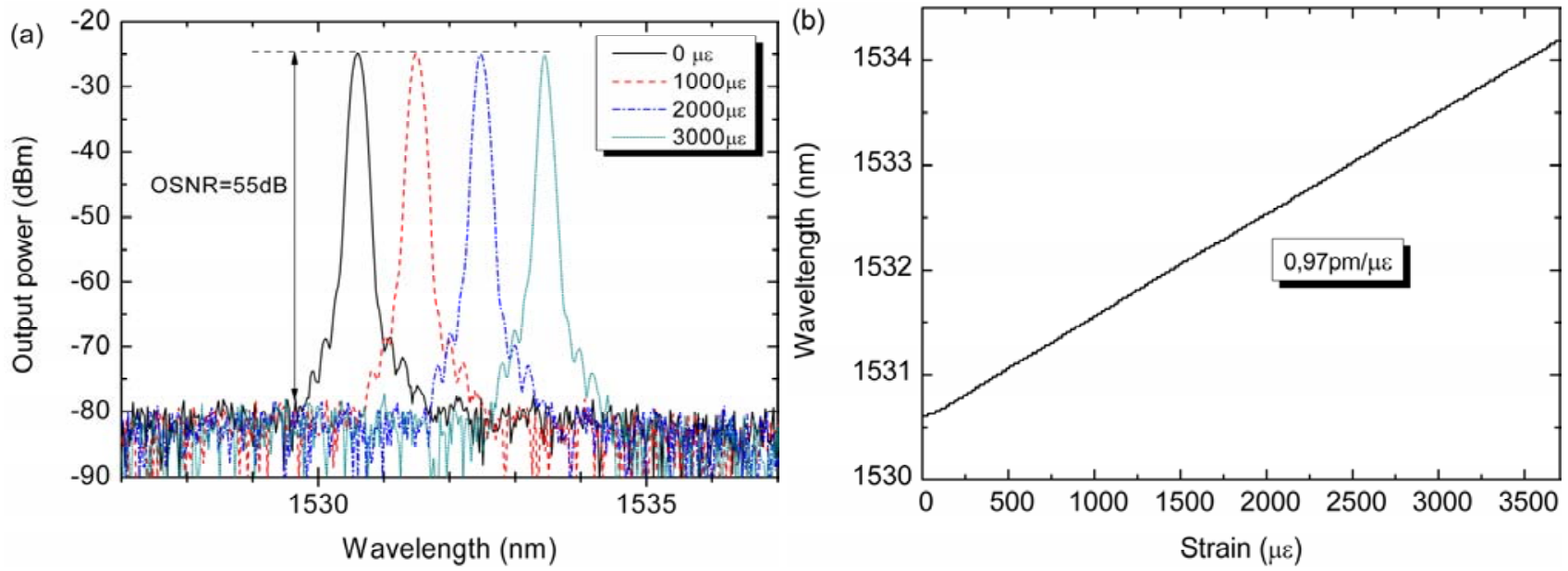

Figure 4. Output spectra of the low-strain sensor for different strain values (a) and, output wavelength evolution with the strain (b).

Finally the transient switching response of the sensors was also studied. This switching time is defined as the time delay between the moment when a sensor is turned off and the next one shows a stabilized output. For the measurements we employed a photo-detector (PDA400 provide by Thorlabs) and an oscilloscope (Agilent 54622A). Figure 5 shows the switching time measured time for B1 to B2 output (solid line) and for B2 to B1 output (dotted line). For both the switching time were less than $250 \mathrm{~ms}$.

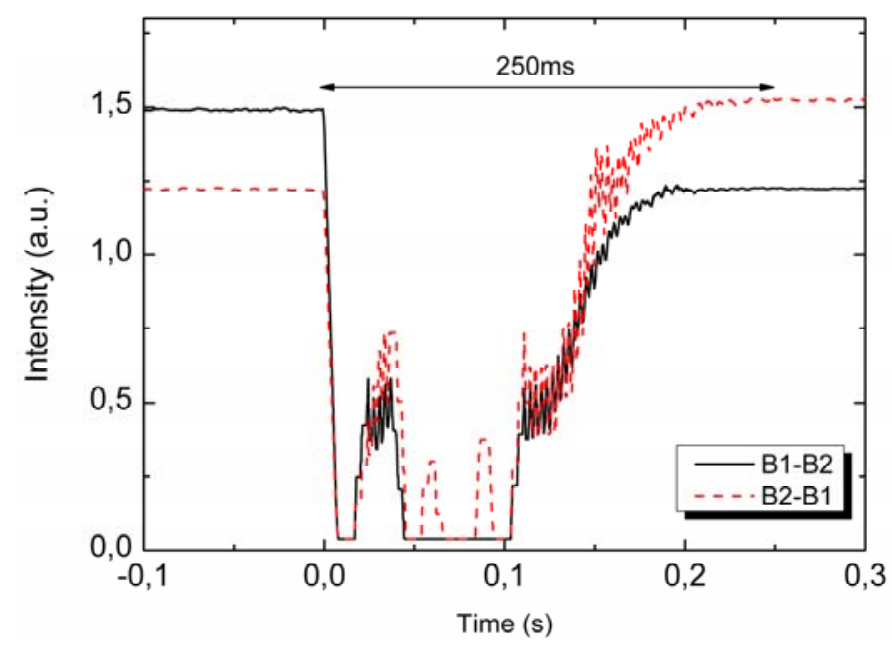

Figure 5. Switching time from B1 to B2 output (solid line) and from B2 to B1 output (dotted line) 


\section{CONCLUSIONS}

To summarize, we propose and demonstrate experimentally a $50 \mathrm{Km}$ switchable fiber optic laser system for fiber optic sensors remote multiplexing. The system allows the integration of different interrogation methods based on the detection of wavelength or amplitude variations of the laser. This integration is sequentially carried out through an optical switch that also allows the use of closer FBG wavelengths for all the sensors. Using this technique, no gain competition among different wavelengths, as happens in multiwavelength lasers, occurs and the utilized wavelength can be always the corresponding to the higher gain of the optical amplifier, in order to optimize the achieved distance of the system. A bending intensity sensor for high strain measurements and a FBG based low-strain sensor were successfully tested. This system includes all the active elements inside the monitoring station, enabling the remote monitoring of the sensors.

\section{ACKNOWLEDGEMENTS}

The authors are grateful to the Spanish government project TEC2010-20224-C02, to the Spanish Ministry of Education and Culture, and to the grant AP2009-1403.

\section{REFERENCES}

[1] Culshaw, B. and Kersey, A. , "Fiber-Optic Sensing: A Historical Perspective" J. Lightwave Technol. 26(9), 1064-1078 (2008).

[2] López-Higuera, J. M., Cobo, L. R., Incera, A. Q. and Cobo, A. , "Fiber optic sensors in structural health monitoring," J.Lightwave Technol. 29(4), 587-608 (2011).

[3] Bravo, M., Saénz, J., Bravo-Navas, M. and López-Amo, M. , "Concrete beam bending test monitorization using a high strain fiber optic sensor," J. Lightwave Technol. 30(8), 1085-1089 (2012).

[4] Bravo, M. and López-Amo, M. , " Remote-Time Division Multiplexing of Bending Sensors Using a Broadband Light Source, " Journal of Sensors. 2012, ID 154586 (2012).

[5] Lee, B. C. and Jeon, M. Y., "Remote fiber sensor based on cascaded Fourier domain mode-locked laser," Opt.Commun. 284(19), 4607-4610 (2011).

[6] Bravo, M., Fernández Vallejo, M. and Lopez-Amo, M. , "Hybrid OTDR-fiber laser system for remote sensor multiplexing," IEEE Sensors Journal 12(1), 174-178 (2012).

[7] Bellemare, A. , "Continuous-wave silica-based erbium-doped fibre lasers," Progress in Quantum Electronics 27(4), 211-266 (2003).

[8] Perez-Herrera, R. A., Diaz, S. , Fernandez-Vallejo, M., Lopez-Amo, M., Quintela M. A. and Lopez-Higuera J. M. , "Switchable multi-wavelength erbium-doped fiber laser for remote sensing," Proc. SPIE 7503, paper $75031 Y(2009)$

[9] Yamashita, S. and Hotate, K. , "Murtiwavelength erbium-doped fibre laser using intracavity etalon and cooled by liquid nitrogen," Electron.Lett. 32(14), 1298-1299 (1996).

[10] Shahi, S., Harun, S. W., Sulaiman, A. H., Thambiratnam, K. and Ahmad, H. , "Multiwavelength source based on SOA and EDFA in a ring-cavity resonator," Microwave Opt Technol Lett 51(1), 110-113 (2009).

[11] Masuda, H. and Kawai, S. , "Wide-band and gain-flattened hybrid fiber amplifier consisting of an EDFA and a multiwavelength pumped Raman amplifier," IEEE Photonics Technology Letters 11(6), 647-649 (1999).

[12] Lee, J. H., Chang, Y. M., Han, Y. -., Chung, H., Kim, S. H. and Lee, S. B. , "Raman amplifier-based longdistance remote, strain and temperature sensing system using an erbium-doped fiber and a fiber Bragg grating," Optics Express 12(15), 3515-3520 (2004).

[13]Fernandez-Vallejo, M. and Lopez-Amo, M. "Optical Fiber Networks for Remote Fiber Optic Sensors" Sensors 12(4), 3929-3951 (2012).

[14] Shih, M. C., Ko, C. L. and Yang, C. Y., "Dynamic strain monitoring by wavelength locking between two fiber Bragg gratings fiber sensing system," Optics and Lasers in Engineering 46(7), 546-549 (2008). 